\title{
Diffuse reflection of phonons and the anomalous Kapitza resistance
}

\author{
Peter Taborek and David L. Goodstein \\ California Institute of Technology, Pasadena, California 91125 \\ (Received 14 February 1980)
}

\begin{abstract}
High-resolution time-of-flight phonon-reflection spectra in sapphire crystals are analyzed with the aid of detailed numerical calculations. It is argued that spectra from vacuum interfaces may be understood if about half the incident phonons are assumed to be reflected specularly, the remainder diffusely. A simple model of the effect on spectra of diffuse scattering is presented and compared to experimental data., We show that the specularly reflected signal is unaffected by placing helium at the interface, but that the diffuse part is transmitted almost entirely, accounting for the anomalous Kapitza resistance. It is shown that conventional calculations of phonon focusing are not suitable for interpreting our data, and a new, more correct theory of phonon focusing, aided by formal catastrophe theory, is introduced. We argue that the experimental resolution and theoretical analysis we have presented are essential for understanding phonon-reflection experiments.
\end{abstract}

\section{INTRODUCTION}

For about a decade, experiments have been reported in which a pulse of thermal phonons propagates ballistically through a pure crystal at low temperature. At the far surface, part or all of the phonons are reflected, and return to be detected as a function of time of flight. The primary purpose of these experiments has been to measure the reflection coefficient of phonons which impinge on a crystal-helium interface; the small reflection coefficient of ballistic phonons is thought to be a manifestation of the well-known anomalous Kapitza thermal boundary resistance.

Although this type of experiment has yielded a number of important insights into the nature of the Kapitza resistance, we wish to suggest that the time-of-flight resolution in most previous experiments has been so low that crucial aspects of the phenomena involved have been obscured or misinterpreted. In this paper, we present an analysis of our high-resolution phonon-reflection timeof-flight spectra which utilizes detailed numerical calculations of phonon trajectories and intensities in the crystal. The result is a far more complete understanding than has previously been possible of the phenomena occurring in these experiments.

Figure 1, from our own early data, is typical of low-resolution spectra. Three broad peaks are seen in the reflected signal. The first and last are, respectively, due to longitudinal and transverse phonons. The intermediate peak is due to phonons that changed polarization upon reflection, making one traversal of the crystal as longitudinal, the other as transverse. In that experiment, as in all the experiments reported in this paper, the sample is a cylindrical sapphire single crystal, with evaporated Ohmic heater(s) and a superconducting-transition bolometer on one face, which is kept in a regulated superfluid bath. The other face is sealed into a cell, where it may be either kept in vacuum, or covered with liquid helium. Both faces are optically polished.

By contrast, a high-resolution spectrum is shown in Fig. 2. The experimental techniques employed to obtain high-resolution spectra have been described in a previous paper. ${ }^{1}$ In that paper we

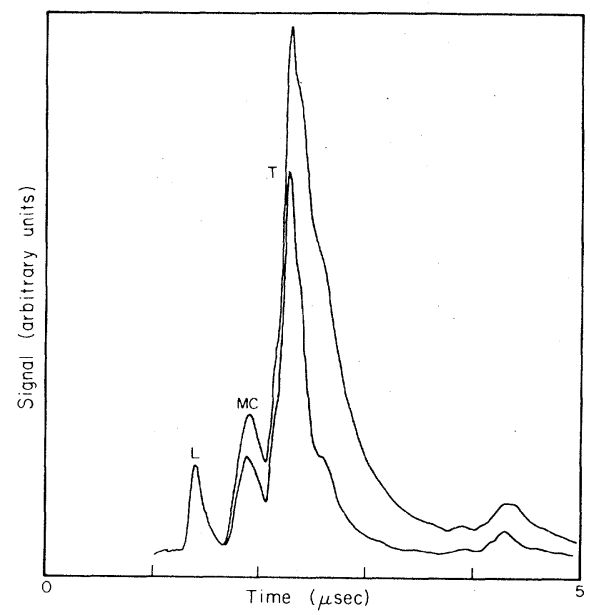

FIG. 1. Typical low-resolution phonon-reflection signal, showing peaks corresponding to longitudinal (L), transverse (T), and mode-conversion (MC) processes. Upper curve is the reflection signal from a vacuum interface, while lower curve is the signal from a crystalliquid-helium interface. 


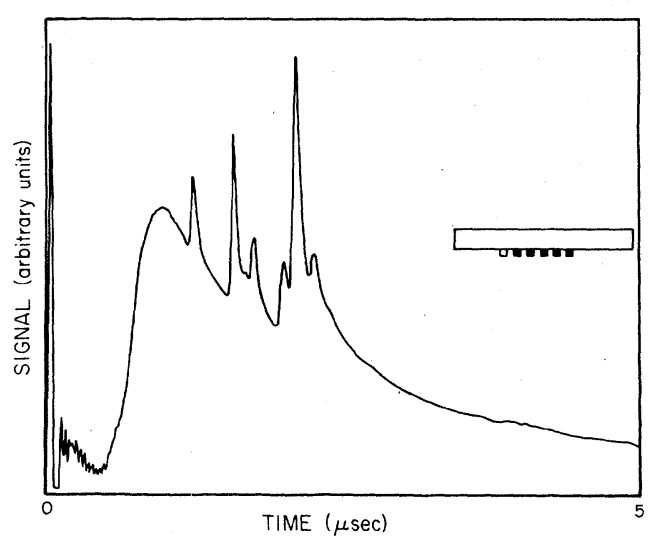

FIG. 2. High-resolution phonon-reflection signal for an unoriented sapphire crystal $6 \mathrm{~mm} \times 57 \mathrm{~mm}$ diameter. The signal is due to firing the middle of the five heaters shown in the inset. The heater-bolometer separation is $6.7 \mathrm{~mm}$; isotropic elastic theory predicts a signal like that shown in Fig. 1. In this and all subsequent spectra, the heater pulses were of approximately 30 -nsec duration, $8 \mathrm{~V}$ in height into a $50-\Omega \mathrm{Al}$ heater at temperature $2 \mathrm{~K}$.

showed there could be as many as nine separate peaks due to specular reflection of phonons, and described how their times of flight could accurately be predicted by numerical techniques. This paper is devoted to analyzing the shapes of those peaks, and other features of the reflection spectra. The arguments we present here will seek to establish the following points:

(1) Of the phonon energy incident on the reflecting surface, in vacuum, some fraction (roughly half in our own experiments) is reflected specularly. Adding helium to the interface never has any detectable effect on those phonons. Their behavior is in full agreement with classical elastic theory. They do not participate in the anomalous Kapitza effect.

(2) The remainder of the phonons, at a vacuum interface, are reflected in some other way. It is consistent with all of our observations to assume they are reradiated with isotropically distributed $\overrightarrow{\mathrm{k}}$ vectors back into the crystal. When helium is added to the interface, these phonons are largely, perhaps almost entirely, transmitted across the interface. They are responsible for the anomalous Kapitza effect.

(3) In certain crystal orientations, phonon-reflection spectra are strongly influenced by the phenomenon known as phonon focusing. Previous theories of phonon focusing are numerically complicated and cumbersome to apply for general directions in crystals. A simpler, and in principle more correct method of computing the phonon in- tensity is presented, and we show how it leads to an understanding of our results.

(4) As a result of the above points, the outcome of a phonon-reflection experiment depends, often critically, on details of the geometry of the experiment which sometimes were not even reported in previous work. We consequently believe that much of the prior work done in this field needs to be reinterpreted or possibly repeated in light of these new arguments.

The remainder of this paper is arranged as follows. In Sec. II, we present some simple arguments showing how diffuse scattering at the interface affects a spectrum when strong focusing ef fects are not present. The results are compared to data, permitting an estimate of the fraction of diffuse scattering in our experiments, and showing that the diffusely scattered phonons become transmitted when helium is present. Section III recounts our attempts to account for other important features of our observations by means of conventional phonon focusing calculations. These attempts were not successful. In Sec. IV we intro-* duce a new more correct theory of phonon focusing and show that it does succeed in accounting for our spectra. The geometrical picture of phonon focusing provided by this theory is helpful in understanding many aspects of phonon propagation and reflection experiments. A summary of our arguments and conclusions is presented in Sec. V.

\section{SIMPLE EFFECTS OF DIFFUSE SCATTERING}

It has been observed in many experiments $\mathrm{s}^{2-4}$ that peaks in the reflection signal due to specular reflection of transverse phonons are often followed by straggling tails; this effect is particularly visible in the last peak in the high-resolution spectrum shown in Fig. 3. The tails represent energy arriving at a time that cannot be accounted for by any specular reflection process. When helium is introduced, the peak is hardly affected, but the tail almost vanishes. Thus the tails clearly arise from processes that occur at the interface and cannot be explained as an artifact due to bulk scattering or slow detector response. In this section we show how the tails and other features of the data may be accounted for by a model based on the picture of diffuse scattering outlined in Sec. I.

Although the details of the diffuse scattering from an optically polished crystal surface are undoubtedly very complicated, we have found that many aspects of the experimental data can be explained by assuming that a constant fraction $\eta$ of the radiation incident at the surface is reradiated uniformly into all solid angles, while fraction $(1-\eta)$ reflects specularly. For mathematical con- 


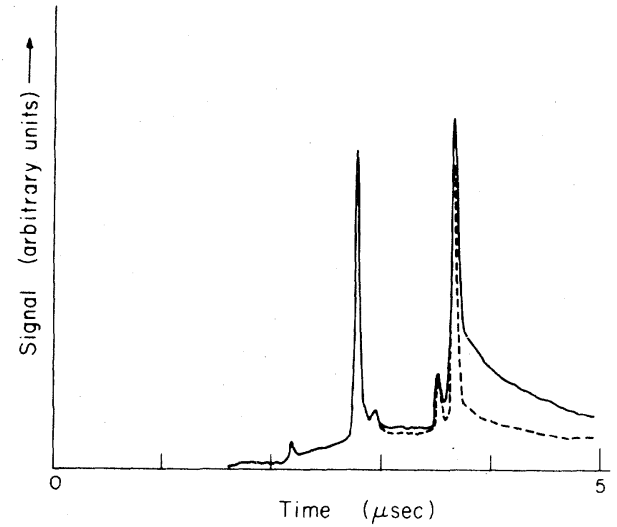

FIG. 3. Reflection signals showing tails. Note especially the last peak. The solid curve is the crystalvacuum interface spectrum, while the dotted curve is the signal from a crystal-liquid helium interface. Note that the large mode-conversion peak at $2.8 \mu$ sec which is well separated from the diffuse scattering signal is not affected at all by the addition of helium. This spectrum was made in a crystal $9.53 \mathrm{~mm}$ thick $\times 12.6 \mathrm{~mm}$ diameter, with heater and bolometer separated by 12.6 $\mathrm{mm}$ along the $C$ axis. Reflection is from the $C X$ plane. See Ref. 1 for further details.

venience, we will ignore the effects of crystal anisotropy in this section and assume that energy flows in the direction of the phonon $\vec{k}$ vector. We will also assume that the heater and bolometer are very small and very close together and can be treated as coincident points.

Since the bolometer signal is proportional to the heat flux, the diffuse scattering signal is the rate at which phonons are scattered back to the detector. The geometry of the calculation is illustrated in Fig. 4, where $h$ is the thickness of the crystal, $c$ is the velocity of sound, and $r$ and $\phi$ are the polar coordinates on the reflection surface; $t$ is the time after the heater pulse, and $Q(t)$ is the heat flux $(\mathrm{J} / \mathrm{sec})$ emitted by the heater as a function of time.

Each element of area $d A=r d r d \phi$ on the top surface is irradiated by phonons from the heater which arrive at $t=\left(r^{2}+h^{2}\right)^{1 / 2} / c$. If each element absorbs the fraction $\eta$ of the incident energy and reradiates it, each area element can be considered as a new source of strength $d Q_{\text {dif }}$ :

$$
d Q_{\mathrm{dif}}(t)=\frac{\eta \cos \theta Q\left[t-\left(r^{2}+h^{2}\right)^{1 / 2} / c\right]}{r^{2}+h^{2}} r d r d \phi .
$$

The diffuse signal $S(t)$ observed at the bolometer is due to the sum of all these elementary sources, and is given by (a)

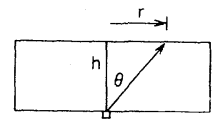

(b)

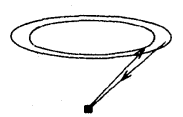

(c)

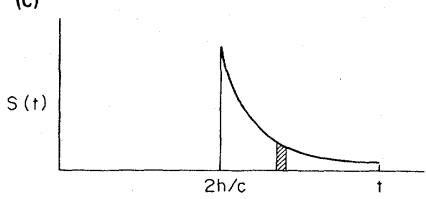

FIG. 4. (a) The geometry for the diffuse signal calculation. (b) The contribution to the diffuse signal at a time $t$ comes from a circular annulus on the reflection surface. (c) A plot of the signal expected from diffuse scattering. The signal has a sharp onset at the time that a specular signal would arrive $t=2 h / c$. The crosshatched regions represents the diffuse signal from points inside the annulus.

$$
S(t)=\int_{\substack{\text { al1 } \\ \text { sources }}} \frac{d Q_{\text {dif }}\left[t-\left(r^{2}+h^{2}\right)^{1 / 2} / c\right]}{r^{2}+h^{2}} \cos \theta
$$

Substituting $\cos \theta=h /\left(r^{2}+h^{2}\right)^{1 / 2}$, and performing the angular integration gives

$$
S(t)=2 \pi \eta h^{2} \int_{0}^{\infty} \frac{Q\left[t-2\left(r^{2}+h^{2}\right)^{1 / 2} / c\right]}{\left(r^{2}+h^{2}\right)^{3}} r d r .
$$

If we assume a delta-function heater pulse $Q(t)$ $=Q_{0} \delta(t)$, the remaining integral can be evaluated explicitly to yield

$$
S(t)=\left\{\begin{array}{l}
0, \quad t<2 h / c \\
2 \pi \eta h^{2} Q_{0}(2 / c)^{4}\left(1 / t^{5}\right), \quad t>2 h / c .
\end{array}\right.
$$

The diffuse signal rises abruptly to its maximum value of $Q_{0} \pi \eta c / h^{3}$ at $t=2 h / c$, the arrival time of the specularly reflected pulse, and then decays as $t^{-5}$ as shown in Fig. 4. The diffuse-signal pulse shape is essentially the result of two competing geometrical effects: For large times the area of the annulus on the reflecting surface which contributes to the signal becomes larger, but the signal is nevertheless small because of the two factors of (distance $)^{-2}$. which account for the decreasing intensity of the incident and scattered energy. In view of the simple model used, this pulse shape seems to be in reasonable agreement with the experimentally observed tails. This calculation also indicates that a tail due to nonspecular scattering has a rather sharp maximum at the same arrival 
time as the specular pulse, so it is intrinsically difficult to distinguish between them experimentally. Numerical calculations show that the sharp maximum at the specular arrival time and the asymptotic decay as $t^{-5}$ remains valid even if the heater and bolometer are separated by a finite distance.

In the model described above, there is only one free parameter, $\eta$, the fraction of nonspecular scattering. $\eta$ can be extracted from the experimental data by comparing the total energy which is scattered back to the detector in the specular and nonspecular channels. The total energy in the specular channel is the area under the specular peak. For a delta-function heat pulse $Q(t)=Q_{0} \delta(t)$, the integral of the specular signal is

$$
E_{\text {spec }}=\int S_{\text {spec }}(t) d t=\frac{Q_{0}(1-\eta)}{4 h^{2}} .
$$

The total energy $E_{\text {dif }}$ which arrives at the detector via nonspecular channels is

$$
E_{\mathrm{dif}}=\int S_{\mathrm{dif}}(t) d t=\eta 2 \pi Q_{0} h^{2}\left(\frac{2}{c}\right)^{4} \int_{2 h / c}^{\infty} \frac{d t}{t^{5}}=\frac{\eta Q_{0} \pi}{2 h^{2}} .
$$

The ratio of the specular to diffuse energy depends only on the fraction of diffuse scattering:

$$
\frac{E_{\text {spec }}}{E_{\text {dif }}}=\frac{(1-\eta)}{\eta} \frac{1}{2 \pi}
$$

This ratio can also be obtained from the experimental data. The only difficulty is in clearly separating the specular and diffuse signal close to the specular arrival time. If the pulse shape of the diffuse signal is extrapolated back to the onset of the specular pulse, the area under the respective curves can be obtained by counting squares. When this procedure is applied, for example, to the last peak in Fig. 3, we find $\eta \simeq 0.5$, a result typical of all our data. The amount of energy which arrives at the bolometer via nonspecular channels is about 6 times greater than the specularly reflected energy, but this is due to geometrical factors. The specular contribution comes from a single small spot on the reflection surface. Although the entire reflection surface contributes to the diffuse signal, the region close to the specular spot is most important.

In summary, then, our picture that a fraction $(1-\eta)$ of the incident energy is reflected specularly, the remaining $\eta$ being scattered isotropically, leads us to expect a sharp specular peak (experimental width $\sim 50 \mathrm{nsec}$ ) superimposed on a tail like that shown in Fig. 4. We do indeed see that behavior, for example in Fig. 3, in Fig. 17 below, and in many other cases, when the surface is in vacuum. These observations indicate that even the simple problem of phonon reflection from a crystal-vacuum interface cannot be adequately described by a model which only considers specular reflection.

The importance of the diffuse component of the signal is even more evident when one considers reflection from a crystal-helium interface. In all cases, the part of the signal we interpret to be due to diffuse scattering is drastically reduced when liquid helium is added to the cell. Although the specular peak is also usually observed to decrease when helium is added, we believe that this apparent decrease is entirely due to the removal of the superimposed diffuse signal. When the overlap of the diffuse tail and the specular peak is properly accounted for, all of our data are consistent with the interpretation that the specularly reflected phonons are not affected by the addition of helium at all. According to classical elastic theory, the acoustic mismatch between sapphire and liquid helium is so severe that we should not be able to detect any change in the signal in these experiments when helium is added to the cell. Thus, we believe that the specular part of the signal obeys elastic theory, and it is the diffusely scattered phonons which are solely responsible for the anomalous Kapitza effect.

That point of view is supported by a number of phenomena observed in our data. Specular peaks which are well resolved from a superimposed diffuse signal are observed to change very little or not at all when helium is added. Figure 3 shows a transverse - longitudinal mode-conversion peak which has very little diffuse background and is unaffected by the presence of helium on the reflection surface. This is in contrast to most previous ly published experiments ${ }^{5-7}$ which report large changes in the mode-conversion peak. We believe that this discrepancy is due to the poor resolution of previous experiments and the failure to distinguish between specular and diffuse scattering. It should be emphasized that the null effect of helium on the mode-conversion peak in Fig. 3 is not due to any special surface preparation. Figure 5 shows a spectrum in which the mode-conversion peaks are accompanied by a diffuse background; in this case helium yields a noticeable effect, although the peak heights actually decrease by less than $10 \%$. The last transverse peak has a substantial tail which decreases monotonically with increasing helium-gas pressure (and therefore increasing liquid-helium film thickness). We have never observed the time-delay phenomena described in Ref. 8. In all of our spectra the effect of helium on the last-arriving transverse peak and the last mode-conversion peak is greater than on 


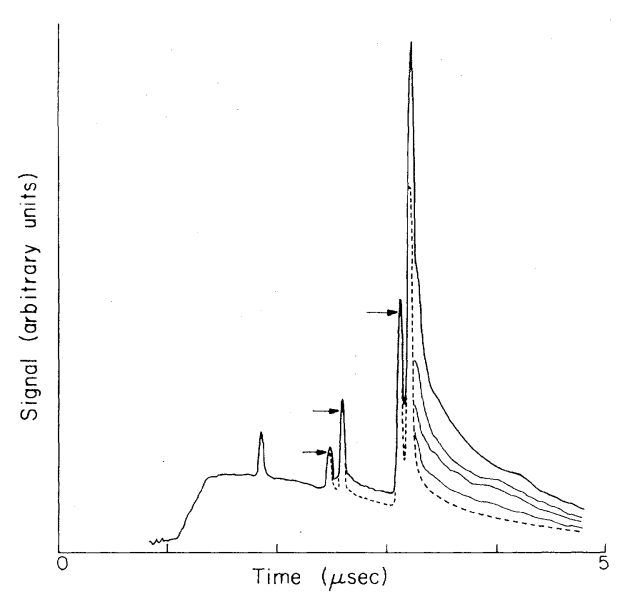

FIG. 5. Spectrum showing a helium effect in the modeconversion peaks (between 2 and $3 \mu \mathrm{sec}$; arrows show peak maxima in bulk helium). Heavy curve: Vacuum interface. Dashed curve: Bulk He. Light curves: He partial saturation, $p / p_{0}$, respectively $6 \times 10^{-5}, 3 \times 10^{-4}$, and $3 \times 10^{-3}$. Experimental arrangement as in Fig. 3 except that heater-bolometer separation was $6.7 \mathrm{~mm}$.

the preceding peaks as shown for example in Fig. 6. We believe that this is due to the fact that a later-arriving specular peak is riding on top of the diffuse tail of the preceding peaks. The addition of helium eliminates the diffuse tails, and the apparent effect on the last specular peak is large. Another feature which we observe in all our data is that the longitudinal peak is never followed by a diffuse tail, and the longitudinal peak is always unaffected by helium. The systematic correlation between the effect of helium on the specular peaks and the presence of an underlying diffuse tail supports our contention that the only effect of adding helium is to remove the diffuse component of the signal; the specular peaks appear to decrease only if the associated diffuse tail decreases.

Another relationship which can be derived from the diffuse scattering model is the scaling behavior of the specular and diffuse signals as a function of the crystal thickness. The time integral of both signals scales as $h^{-2}$, as one would expect, but the maximum of the nonspecular signal scales as $h^{-3}$. The ratio of the specular to the diffuse-scattering signal at $t=2 h / c$, the specular pulse arrival time,

$$
\frac{S_{\mathrm{spec}}(2 h / c)}{S_{\mathrm{dif}}(2 h / c)} \propto h,
$$

increases linearly with $h$. Thus, experiments with long crystals allow a better separation of specular and diffuse scattering than short ones.

Among our earliest experiments were a series

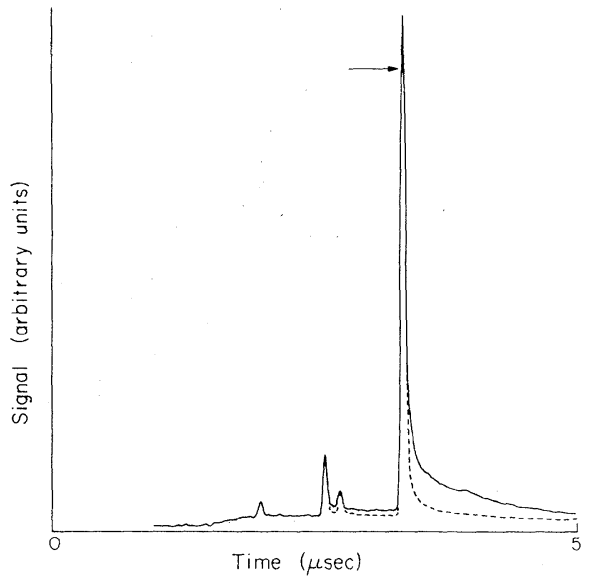

FIG. 6. Spectrum showing that the helium effect increases with each successive peak. Experimental arrangement as in Figs. 3 and 5 except that heater-bolometer separation was $9.3 \mathrm{~mm}$.

performed in a long crystal $(42 \mathrm{~mm} \times 22 \mathrm{~mm}$ diameter). To our considerable surprise, the peaks in the spectrum seemed to be affected only slightly by adding helium to the vacuum interface. We now understand that to have been a result of length scaling. It has recently been reported that in situ cleaving can eliminate the helium effect on reflection spectra. ${ }^{9}$ We have here another way to achieve that result. In fact, the question of whether and how the anomalous Kapitza effect is to be observed in a given spectrum always depends on details of the geometry of the experiment. It may be larger or smaller than one might naively expect. In some crystallographic orientations of heater and bolometer, the effect of helium on the reflection signal can be remarkably large, as is discussed in the following sections.

\section{CONVENTIONAL PHONON FOCUSING AND DIFFUSE SCATTERING}

The spectrum shown in Fig. 7 has six sharp peaks, each at the predicted arrival time for one or more specular reflection channels. Following the last of these six peaks, there is a large bump, somewhat broader than the specular peaks. The bump does not correspond to the time of arrival of any specular process, but it is energy that returns from the reflecting surface in a narrow, concentrated period of time. We have observed many features of this kind, some of them much larger than the one in that spectrum. We shall show that these bumps are due to diffusely scattered radiation which goes from heater to surface to detector along intense directions of phonon focusing. How - 


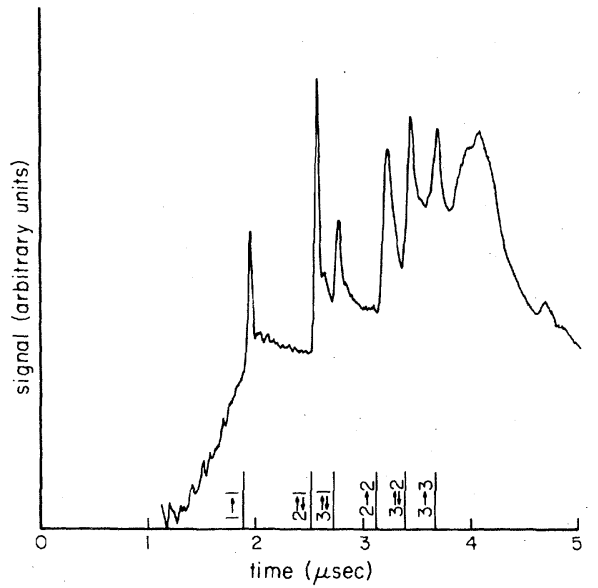

FIG. 7. Spectrum showing peaks due to various reflection processes. Polarizations are labeled $1,2,3$ in order of wavespeed, arrows indicating the change in polarization on reflection. Labels refer to lines along the time axis showing arrival times predicted by numerical calculations from elastic theory. See Ref. 1 for details. Arrangement differs from that of Figs. 3, 5, and 6 in that heater and bolometer are separated by $9.0 \mathrm{~mm}$, this time along the $X$-axis. Note the large bump after the last $(3 \rightarrow 3)$ predicted peak.

ever, our initial attempts to identify the source of these features using standard technique ${ }^{10,11,3}$ to analyze the effects of phonon focusing proved futile. We were able to deduce the origins of the bumps only after developing a new, more correct theory of phonon focusing. Before introducing the new analysis, we wish to recount our efforts to use conventional techniques in order to demonstrate their inadequacy.

Phonon focusing occurs in all real crystals as a consequence of crystal anisotropy. Because the $\overrightarrow{\mathrm{k}}$ vector and the energy flux or Poynting vector $\overrightarrow{\mathrm{p}}$ of a phonon in a crystal are not collinear, the energy flux emitted from the heater is not uniform even if, as we assume, the $\vec{k}$ vectors of the emitted phonons are uniformly distributed. Phonon focusing influences the amount of energy which reaches a given point on the surface from the heater, as well as the intensity of scattering from this point in the direction of the detector.

Crystal anisotropy also influences the diffusely scattered part of the spectrum in another way. In the isotropic case, the contribution to the diffuse signal between $t$ and $t+\Delta t$ comes from a circular or elliptical ring on the reflection surface (see Fig. 4). In a real crystal, however, the set of points on the reflection surface which have total flight time $t$ from heater to detector via the surface is some irregular curve that has no convenient analytic expression.
In order to take these effects into account and to identify the position on the reflection surface which was responsible for bumps like that in Fig. 7 , we set out to make contour plots of the energy distribution on the reflection surface and the flight time for each mode. By superimposing these contour plots, we hoped to examine the intensity on the reflection surface at points which had flight times which corresponded to the large diffuse peaks. Unfortunately, severe difficulties arose in carrying out that project.

The contour plots were constructed by interpolating values of the intensity and time of flight from a $20 \times 20$ table of computed values. This grid of 400 points represented a $4-\mathrm{cm}^{2}$ area on the reflection surface centered above the heater. Both the intensity and the time of flight depend on the Poynting vector $\vec{p}$, which in turn depends in a complicated way on the $k$ vector and polarization. We have previously described similar calculations for specular reflection. ${ }^{1}$ For each point on the grid, it was first necessary to find the $k$ vectors which corresponded to the Poynting vectors which cause energy to flow from the heater to the grid point and then back to the detector. This involves iterative calculations similar in principle to those for specular reflection. ${ }^{1}$ Since the $\vec{k}$-vector calculation had to be performed hundreds of times per contour plot, it was necessary to write an efficient algorithm to find $\vec{k}$ given the direction of $\vec{p}$ in order to keep the computing time within reasonable limits.

By analogy to the specular reflection analysis, one might expect that there are nine processes which connect heater and detector via an arbitrary grid point. Since each of the nine scattering processes has a distinct arrival time, one would expect to require nine separate arrival-time contours for a complete description. In order to simplify the problem, we confined attention to the non-mode-conversion transverse $\rightarrow$ transverse scattering processes, which the experiments indicated were most important.

The apparently simple matter of labeling the transverse modes in an anisotropic solid turns out on closer inspection to present an insoluble difficulty. In Ref. 1 we discussed the disadvantages of the simple (L, FT, ST) phase-velocity labeling scheme. We suggested a labeling method which consisted of choosing a direction in $\vec{k}$ space, labeling the three modes 1 (longitudinal), 2 (fast transverse), and 3 (slow transverse) in order of their speeds in that direction, then continuing the polarizations smoothly along the slowness surface to any direction of interest. The slowness surface is the surface of constant $\omega$ in $\vec{k}$ space representing each of the three modes. Un- 
fortunately, this labeling scheme did not give unique answers when applied to a general grid point. More precisely, the polarization can be continuously transported along a closed path on the slowness surface, and the initial and final polarizations may not be equal. The reason for this behavior seems to be that there exist points where the curves of constant $\omega$ (sections of the slowness surface) for the two transverse modes osculate, as shown in Fig. 8. In a sense, the surfaces touch without crossing. Starting from one curve on one side of the point $A$, one can continue the polarization continuously to either curve on the other side of the point. This seems to mean that there is no way, even in principle, to divide the transverse reflection processes into globally distinct classes which have a continuous variation of some vectorial characteristic of the phonon. The previous success of the method for the analysis of the specular processes is due to the fortunate circumstance that no osculation point was in the vicinity of the connecting paths.

Because of the phonon labeling problem, it is impossible to associate with each grid point the flight time of a given transverse-phonon scattering process; the best that can be hoped for is to associate with each grid point four $\vec{k}$ vectors and four times which correspond to two outgoing (from the heater) and two ingoing transverse phonons. Even this is optimistic, since in fact there may be more than two transverse phonons which cause energy flow between the heater and a given point on the reflection surface.

To see how this can happen, it is useful to remember that the group velocity of a wave packet, which is in the direction of energy flow, is given by $\nabla_{\vec{k}} \omega$. Thus, the normal vectors to the $\omega=$ constant surface, or slowness surface, are parallel to the energy-flux vectors. The problem of finding all phonons that transport energy from the origin to a given point $\vec{x}$ can be interpreted geometrically as finding all points on the slowness surface with

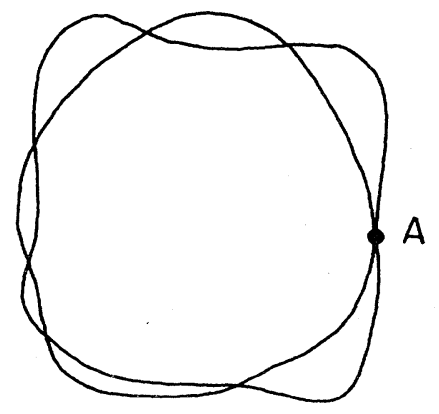

FIG. 8. Schematic diagram showing two sheets of a slowness surface which osculate at point $A$. normal vector parallel to $\vec{x}$. If the slowness. surface is convex like a sphere or ellipsoid, there is only one solution to the problem. If, however, the surface is more complicated, multiple solutions arise as shown in Fig. 9. The figure shows three distinct $\overrightarrow{\mathrm{k}}$ vectors which yield normals and energy flow in precisely the same direction, although the magnitudes of the three group velocities are not obliged to be equal. The far field due to an instantaneous point source may be quite complicated in an anisotropic medium; instead of observing three pulses corresponding to longitudinal, fast transverse and slow transverse, in some directions one may observe several pulses from each polarization. ${ }^{12}$ It appears that in sapphire the longitudinal sheet of the slowness surface is actually convex, so multiple solutions occur only for the transverse modes. It is easy to convince oneself that multiple solutions for the transverse branches are not confined to special high-symmetry points, but occur in general directions as well. Detailed numerical calculation shows that there are many regions in sapphire in which five or six transverse pulses can be observed.

Before it was fully realized that many $\vec{k}$ vectors might lead to energy propagation in the same direction, the computational procedure for finding the $\vec{k}$ vector which sent energy in the $\vec{x}$ direction was based on using $\vec{k} \| \vec{x}$ as a first guess, and then improving the guess until a solution was found. As can be seen from Fig. 9, this technique would only yield the solution $\overrightarrow{\mathrm{k}}_{2} ; \overrightarrow{\mathrm{k}}_{1}$ and $\overrightarrow{\mathrm{k}}_{3}$ cannot be reached by improving a guess which is close to $\vec{k}_{2}$. Moreover, there is no way to generate a guess $a$ priori which is close to $\overrightarrow{\mathrm{k}}_{1}$ or $\overrightarrow{\mathrm{k}}_{3}$. Mathematically, the problem is to find the global solutions to a coupled set of nonlinear vector equations in vector

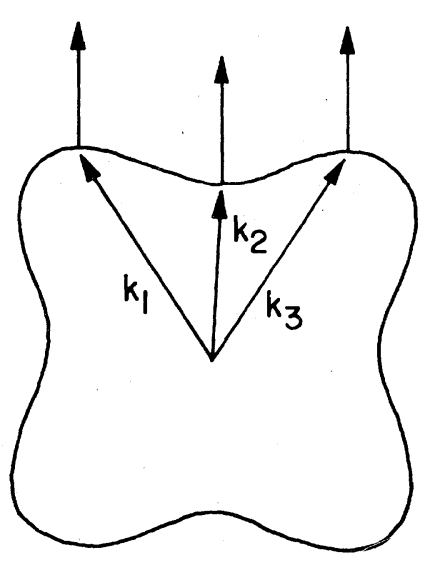

FIG. 9. Schematic diagram of a slowness surface showing three $\vec{k}$ vectors which produce energy flow in the same direction. 
unknowns. Since the only practical way to solve nonlinear equations is to improve a sufficiently good guess, it was necessary to compute the Poynting vectors for a dense grid of $\overrightarrow{\mathrm{k}}$ vectors on the entire slowness surface to find good initial guesses for cases like $\overrightarrow{\mathrm{k}}_{1}$ and $\overrightarrow{\mathrm{k}}_{3}$ of Fig. 9. With these added complications, one must be prepared to associate more than two ingoing and/or outgoing transverse-phonon $\vec{k}$ vectors with each grid point. Once all the $\vec{k}$ vectors have been found, the time of flight can be computed for each process in a straightforward manner.

The computation of the intensity of the wave packet corresponding to each $\vec{k}$ vector involves the theory of phonon focusing. ${ }^{10,11}$ The way elastic anisotropy may focus or defocus energy is illus trated in Fig. 10. A wave packet with $\overrightarrow{\mathrm{k}}$ vectors contained in a solid angle given by $d \vec{\Omega}_{k}=d \overrightarrow{\mathrm{k}}_{1} \times d \overrightarrow{\mathrm{k}}_{2}$ sends energy into a solid angle $d \vec{\Omega}_{p}=d \overrightarrow{\mathrm{p}}_{1} \times d \overrightarrow{\mathrm{p}}_{2}$. In an isotropic solid, the $\overrightarrow{\mathrm{k}}$ and $\overrightarrow{\mathrm{p}}$ vectors are parallel, so $d \vec{\Omega}_{k}=d \vec{\Omega}_{p}$, and there is no focusing. In an anisotropic solid, however, the $k$-vector py ramid and the corresponding Poynting-vector pyramid may have considerably different shape. If $d \Omega_{k}$ $>d \Omega_{p}$, the energy is focused, while if $d \Omega_{k}<d \Omega_{p}$, the energy is spread over a larger area and the intensity is low. The ratio $d \Omega_{k} / d \Omega_{p}$, which is called the focusing factor, can be used to measure the intensity of the energy flux which travels along $\overrightarrow{\mathrm{p}}$. In order to calculate the differential area $d \Omega_{p}$, one must know the change $d \overrightarrow{\mathrm{p}}$ caused by a change $d \overrightarrow{\mathrm{k}}$. This is given by the formula

$$
d p_{i}=\frac{\partial p_{i}}{\partial k_{j}} d k_{j}
$$

where $i$ or $j=x, y, z$ and we use the summation convention. Because of the dependence of $p_{i}$ on the polarization which in turn depends on $\overrightarrow{\mathrm{k}}$, the derivative $\partial p_{i} / \partial k_{j}$ is rather complicated. First, the $k$ derivative of the polarization must be computed. The result is ${ }^{11}$

$$
\frac{\partial e_{k}^{\gamma}\left(k_{j}\right)}{\partial k_{i}}=\sum_{\alpha \neq \gamma} \frac{1}{\rho}\left(c_{i j l m}+c_{i l j m}\right) \frac{e_{i}^{\alpha} e_{m}^{\gamma} e_{k}^{\alpha} k_{l}}{v_{\gamma}^{2}-v_{\alpha}^{2}},
$$

where $e_{i}^{\alpha}\left(k_{j}\right)$ denotes the $i$ th component of the unit polarization vector of the $\alpha$ mode $(\alpha=1,2,3)$ with
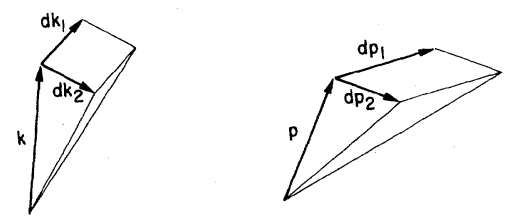

FIG. 10. A wave packet in $\vec{k}$ space and the corresponding Poynting vectors in real space, showing, in this case, phonon defocusing. $k$ vector $k_{j}$, and $v_{\alpha}$ is the corresponding phase velocity. $c_{i j l m}$ is the elastic tensor. Substituting into this expression the formula

$$
\begin{aligned}
\frac{\partial p_{i}^{\alpha}}{\partial k_{i}}= & p_{i}^{\alpha} p_{j}^{\alpha}+\frac{1}{\rho} c_{n j m i} e_{n}^{\alpha} e_{m}^{\alpha} \\
& +c_{n l m i} k_{l}\left(\frac{\partial e_{n}}{\partial k_{j}} e_{m}^{\alpha}+e_{n}^{\alpha} \frac{\partial e_{m}}{\partial k_{j}}\right)
\end{aligned}
$$

yields the required derivative which can be used to compute the focusing factor. A computer program was written to evaluate the various derivatives and the intensity for each $\vec{k}$ vector associated with a grid point. Although these formulas have previously been used to calculate the intensities in high-symmetry directions in cubic crystals, ${ }^{11}$ most published phonon-focusing calculations have used a statistical method which yields only low-resolution information about the relative phonon intensity.

Once the analysis outlined above has been completed, each grid point has associated with it a list of incoming and outgoing intensities and arrival times. In order to construct a contour plot, one phonon process must be selected at each grid point with its corresponding flight time and intensity. Because of the phonon-labeling complications discussed above, there may not be a unique way to make the choice. This is not an insurmountable problem, since the contours will contain information of interest as long as they are reasonably continuous and smooth. It was found that smooth contours could be obtained by choosing the two processes with highest intensity and labeling them according to the magnitude of the group velocity as fast transverse and slow transverse.

A typical set of contour plots which results from this analysis are shown in Figs. 11 and 12.

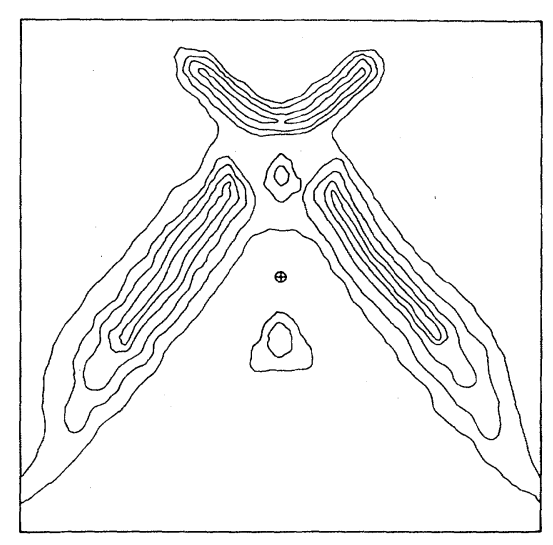

FIG. 11. Contours of constant intensity on the surface of a sapphire crystal. Heater is below the spot marked $\oplus$. Surface normal is the $Y$ axis. See text for details. 


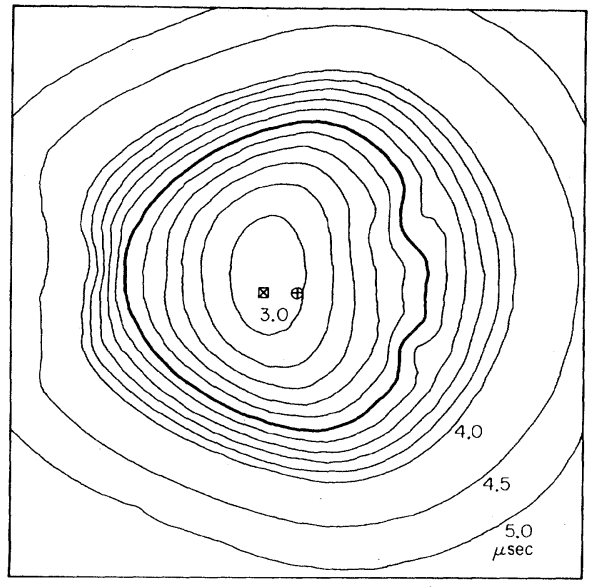

FIG. 12. Contours of constant arrival time corresponding to intensities in Fig. 11. Heater below $\oplus$, detector below $\otimes$.

Figure 11 shows the intensity distribution of the fast transverse mode on the upper surface of the sapphire crystal. The rather dramatic effects of elastic anisotropy on energy flow in the crystal can be clearly seen. Figure 12 shows the contours of constant arrival time for all scattering processes that involve ingoing and outgoing fast transverse phonons for the experimental geometry corresponding to the reflection signal shown in $\mathrm{Fig}$. 16 below. The arrival time of the large nonspecular bump in Fig. 16, 3.5 $\mu \mathrm{sec}$, is marked by the heavy contour. When the arrival time and intensity contour maps are superimposed, the heavy contour overlaps several regions of high intensity, but many other time contours overlap equally intense regions. The large diffuse features seen in Figs. 7 and 16 are apparently not due in any simple way to the fact that the heater emits energy anisotropically.

\section{PHONON CAUSTICS: THEORY AND EXPERIMENT}

During the course of further analysis of intensity contours like Fig. 11, it was discovered that the numerical routines were unstable in some regions of high intensity, and the computer would sometimes converge on isolated points where the focusing factor was very large. Because the Eqs. (10) and (11) for the focusing factor are rather complex, it is difficult to get a physical understanding of why the intensity is much higher in some regions than in others. In an attempt to gain some insight into this problem, we developed a different method of analyzing phonon focusing based on an analysis of the asymptotic field from a point acoustic source. ${ }^{13,14}$ The integrals which arise in the problem are of the type dealt with in formal catastrophe theory, and this theory can be used to make very general statements about the form of regions of high focusing using some simple geometric arguments.

A general expression of the acoustic field at $x$ from a point source at $x^{\prime}$ is obtained by constucting the Green tensor $g_{k m}\left(x \mid x^{\prime}\right)$ which satisfies the anisotropic wave equation with a periodic point source:

$$
L_{i k} g_{k m}\left(x \mid x^{\prime}\right)=\delta_{i m} \delta\left(x-x^{\prime}\right),
$$

where, if $c_{i j l k}$ is the elastic tensor, then

$$
L_{i k}=c_{i j l k} \frac{\partial^{2}}{\partial x_{l} \partial x_{i}}-\omega_{0}^{2} \delta_{i k},
$$

and its Fourier transform is

$$
\tilde{L}_{i k}=c_{i j l k} k_{i} k_{j}-\omega_{0}^{2} \delta_{i k} \text {. }
$$

A similar equation for the far field from a point source in an anisotropic medium is analyzed in Refs. 15 and 16; the treatment here is adapted from those references.

To examine the field at a point $P$ due to a source at the origin, we transform to a frame in which $P$ has coordinates $(0,0, z)$. The solution is

$$
g_{k m}(P \mid 0)=\int \frac{B_{k m}}{\left(\partial G / \partial k_{z}\right)} e^{i k_{z}\left(k_{x}, k_{y}\right) z} d k_{x} d k_{y},
$$

where $B_{k m}$ is the matrix of cofactors of $\tilde{L}_{k m}, G$ $=\operatorname{det} \tilde{L}$ and the integral is over the slowness surface defined by $G=0$ (surface of constant $\omega$ in $\overrightarrow{\mathrm{k}}$ space). Equation (13) represents the field at $P$ in terms of a weighted sum of plane waves which have $\vec{k}$ vectors on the slowness surface. The integral cannot be carried out exactly, but for large $z$ it can be approximated using the principle of stationary phase. The phase is stationary at points where $\left(\partial k_{z} / \partial k_{x}\right)$ and $\left(\partial k_{z} / \partial k_{y}\right)$ are zero; geometrically this represents points on the slowness surface with the normal vector in the $\hat{z} \mathrm{di}-$ rection. Waves with $\overrightarrow{\mathrm{k}}$ vector in the vicinity of the stationary point $\overrightarrow{\mathrm{k}}_{0}=\left(k_{x}^{0}, k_{y}^{0}, k_{z}^{0}\right)$ contribute to the integ ral in Eq. (13), but waves with other $k$ vectors tend to be out of phase and cancel each other. The exponent in Eq. (13) can be expanded to second order around the stationary point, which, in an appropriately chosen coordinate system yields:

$$
k_{z}=k_{z}^{0}+\alpha\left(k_{x}-k_{x}^{0}\right)^{2}+\beta\left(k_{y}-k_{y}^{0}\right)^{2} .
$$

When this is substituted into Eq. (13) and the limits are extended to $\pm^{\infty}$, the leading order behavior of the field is obtained:

$$
g_{k m}(P \mid 0) \propto B_{k m}\left(k_{z}^{0}\right) e^{i k_{z}^{0} z / z \sqrt{\alpha \beta}} .
$$

The product $\alpha \beta$ is the Gaussian curvature of the slowness surface at the point of stationary phase 
$\overrightarrow{\mathrm{k}}^{0}$, and in this approximation, the field decreases as $z^{-1}$ and the intensity is inversely proportional to the Gaussian curvature $\alpha \beta$. We have thus constructed a geometric representation of phonon focusing. In retrospect it is evident that the ratio of solid angles shown in Fig. 10 is formally identical to the curvature of the slowness surface as defined by Gauss.

The geometric measure of focusing has considerable advantages for making qualitative predictions over the traditional approach. For instance, it is clear that directions of high focusing are associated with regions of small curvature on the slowness surface and that points of zero curvature yield an infinity in the field amplitude. An infinite or even very large displacement is of course incompatible with linear elastic theory. This result is due to a breakdown of the geometrical optics approximation that has been made in deriving Eq. (15).

The possible forms of regions of zero curvature can be investigated by considering perturbations of the slowness surface of an isotropic solid, for which each sheet is a sphere in $\vec{k}$ space. The effect of elastic anisotropy is to deform the spheres slightly; this generally (but not inevitably) leads to regions of negative curvature. Regions of positive and negative curvature are separated by smooth curves along which the curvature vanishes.

Vectors normal to the slowness surface along such a closed curve correspond to Poynting vectors which sweep out a (topologically) conical surface emanating from the point source on which the field is intense but cannot be computed using geometrical optics. Such surfaces are known as caustics in classical wave theory, and higherorder approximations to the wave equation must be used to analyze the field in their vicinity. If, for example, $\alpha$ in Eq. (14) is close to zero, the expansion is not sufficiently accurate and another term must be included:

$$
\begin{aligned}
k_{z}= & k_{z}^{0}+\alpha\left(k_{x}-k_{x}^{0}\right)^{2}+\gamma\left(k_{x}-k_{x}^{0}\right)^{3} \\
& +\beta\left(k_{y}-k_{y}^{0}\right)^{2} .
\end{aligned}
$$

The retention of the third-order term is necessary because there are two nearby points where the phase is stationary, corresponding to two parallel rays, as shown in Fig. 13. On the caustic, $\alpha=0$, the rays merge, and the geometrical optics approximation goes to infinity, but the actual field is finite and is given by

$$
g_{k m}(P \mid 0) \propto B_{k m}\left(k_{z}^{0}\right) \frac{e^{i k_{z}^{0} z}}{z^{5 / 6}} \frac{1}{\beta^{1 / 2}} \frac{1}{\gamma^{1 / 3}} .
$$

The intensity is not uniform along the caustic as suggested by the geometrical optics approxima-
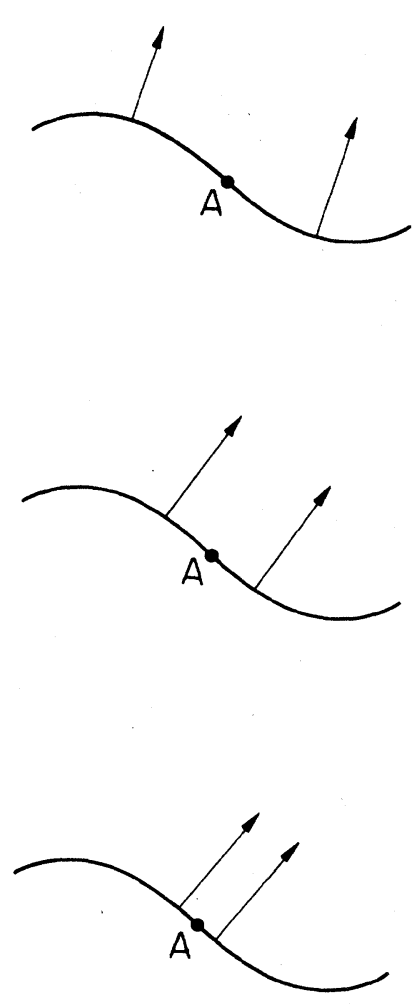

FIG. 13. Section of a slowness surface which contains a point $A$ of zero curvature. On either side of $A$, there are two $\vec{k}$ vectors which yield energy flow in the same direction. As the $\overrightarrow{\mathrm{k}}$ vectors approach $A$, the two rays merge. Note that in the vicinity of $A$, the slowness surface can be approximated by a cubic polynomial-like Eq. (16).

tion, but rather depends on the third derivative term $\gamma$; remarkably, the field also has a $z^{-5 / 6}$ spatial dependence, rather than the $z^{-1}$ dependence normally expected from a point source.

The breakdown of geometrical optics that occurs on a caustic is an example of a catastrophe in the sense of formal catastrophe theory; this point of view is developed in Refs. 17 and 18. It should be emphasized that the catastrophes are not physical ones. In fact, this analysis replaces nonphysical infinities in the old theory by intensities that are finite everywhere. For elastic waves in a solid, catastrophe theory allows only two types of structurally stable caustic behavior. The simplest, known as a fold catastrophe, corresponds to the coalescence of two rays, and is associated with a third-order expansion such as Eq. (16). Figure 13 shows how the rays merge at a simple inflection point. Along the caustic, $\gamma$ may occasionally vanish at isolated points, where a fourth-order expansion is required. These points are known as 
cusp catastrophes (because of the characteristic shape of the caustic) and correspond to the coalescence of three rays. ${ }^{19}$ A simple analysis shows that the field at a cusp has spatial dependence $z^{-3 / 4}$. No catastrophes of yet higher order can exist at a typical point. A summary of the catastrophe theory analysis of caustics in anisotropic media is shown in Fig. 14.

Once it was realized that the intensity distribution on the top surface of the crystal would be dominated by the caustic curves where the focusing factor was infinite, the computer programs used to construct the contour plot of Fig. 11 were rewritten so that the caustics could be located and plotted, as shown in Fig. 15. As expected on the basis of catastrophe theory, cusps where the field is particularly high are a prominent feature in the figure. A comparison of Fig. 11, which was constructed from a table of intensities on a finite grid and Fig. 15 which locates the caustics, shows that the intensity distribution interpolated from the finite grid can be quite misleading; only some of the high-intensity regions visible in Fig. 11 are associated with an underlying caustic, but these are the most important. An important implication of the catastrophe analysis and the structural stability of caustics is that phonon caustics are a typical feature which can be expected to exist in most crystals. The structure of the caustics and the resulting fine structure in the phonon intensity distribution in $\mathrm{Ge}$ has been vividly revealed in a recent experiment. ${ }^{20}$

The caustic structure of the point source can be used to understand the sharp nonspecular features which are observed in some reflection geometries but not in others. It must be kept in mind, however, that in a reflection experiment diffusely scattered phonons can be refocused by the crystal anisotropy. Thus, there are two sets of caustics which are important to describe a reflection experiment: the heater caustics intersect

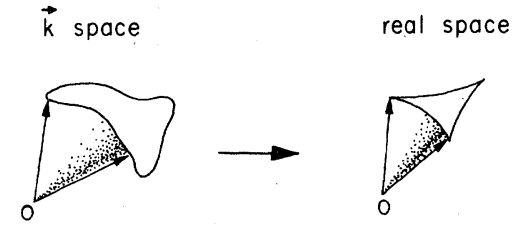

FIG. 14. The $\vec{k}$ vectors which correspond to points of zero curvature on the slowness surface define a topologically conical surface in $\vec{k}$ space which has a smooth boundary, without sharp corners. The Poynting vectors which are associated with the $\vec{k}$ vectors also sweep out a topologically conical surface, but it generally has sharp cusp-like edges.

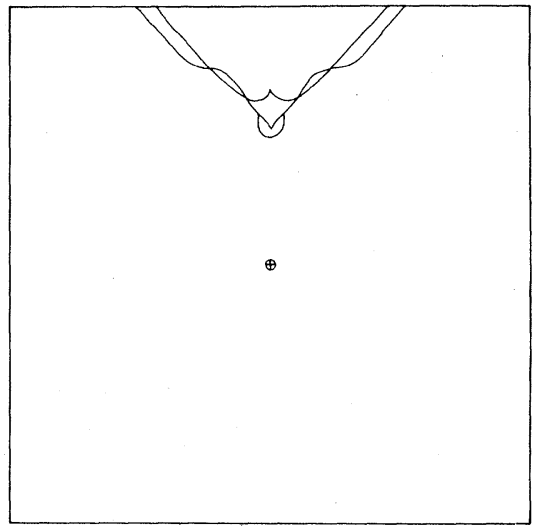

FIG. 15. Location of caustics for both transverse modes in sapphire. The curves are the intersection of the topologically conical caustic surface which emanates from the heater with the upper surface of the crystal. The position of the heater is marked by the circled cross. Compare with Fig. 11.

the reflection surface in curves of high incident intensity, while similar caustics are associated with the detector and can be interpreted as a set of points where a source would focus strongly back to the detector. The insets of Figs. 16 and 17 schematically show the orientation of the heater and detector caustics (detector caustic is dotted) for the two representative experiments. Although the caustics are drawn as lines, they have a width

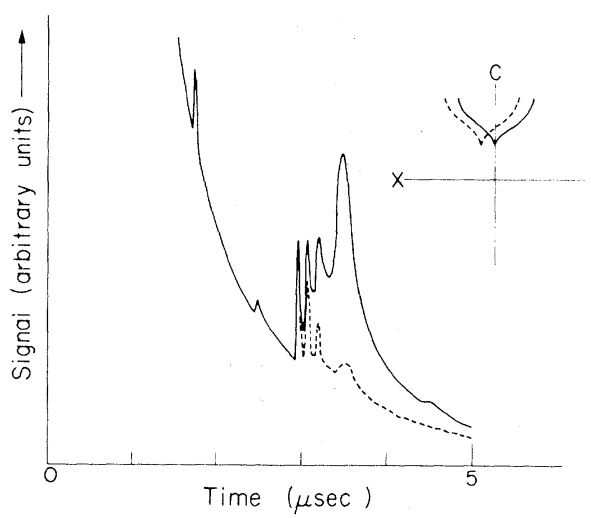

FIG. 16. Reflection signal for heater and bolometer displaced along the $X$ axis ( $Y$ axis is the reflection surface normal). The inset shows the intersection of the heater (solid curve) and bolometer (dotted curve) caustic surfaces with the crystal reflection surface. The intersection of the two caustics provides a high-focusing channel for nonspecular scattering which gives rise to the large bump in the reflection signal. Dashed curve in the main figure shows the effect of adding helium. Experimental arrangement as in Fig. 7, except that heaterbolometer separation is $2.0 \mathrm{~mm}$. 


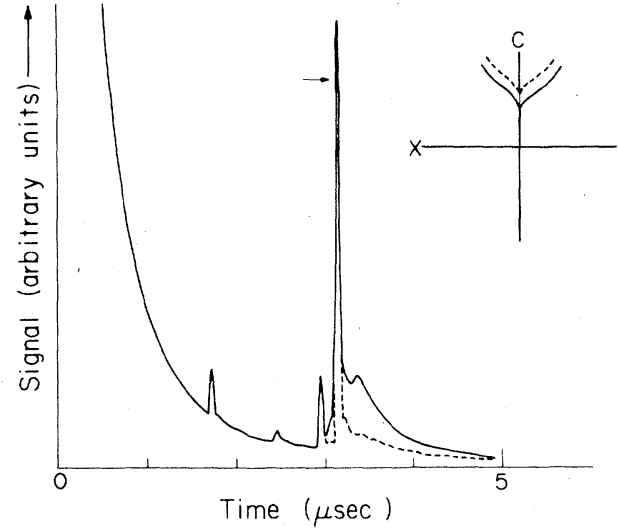

FIG. 17. Reflection signal for heater and bolometer displaced along $C$ axis. The inset shows that for this geometry, the heater and detector caustics do not intersect. The ratio of specular to diffuse scattering is much higher in this geometry than in $X Y$ plane reflection shown in Fig. 14. The dashed curve in the main figure shows the effect of helium. Experimental arrangement as in Figs. 3, 5, and 6 except that heater-bolometer separation was $2.0 \mathrm{~mm}$.

given by the size of the source and detector.

The sapphire crystal is trigonal. It has one three-fold axis which we designate the $C$ axis, and three two-fold axes in the plane perpendicular to $C$. We designate one of the two-fold axes to be $X$, then choose $Y$ so that $X, Y, C$ form a right-handed Cartesian system. All of the samples in the experiments to be described in the remainder of this section are cylinders whose axis is the $Y$ axis of the crystal, and whose faces are therefore in the $C X$ plane.

If the heater and detector are displaced along the $X$ axis, as in Fig. 16, the caustics overlap and there is a spatially localized high-intensity nonspecular scattering channel. The time-of-flight of this process agrees precisely with the arrival time of the large diffuse peak. If the heater and bolometer are placed along the $C$ axis, the caustics do not overlap and there is no prominent peak in the diffuse scattering signal, as shown in Fig. 17.

In both Figs. 16 and 17, the effect of adding helium to the reflecting surface is also shown. In Fig. 17, where there is no nonspecular bump, the behavior is just as we would expect from the arguments of Sec. II. In fact, we have already seen much the same result in Figs. 3, 5, and 6. The reasoning that led us to expect that result arose from the assumption that radiation that is diffusely scattered at the vacuum interface would disappear into the helium when helium is present. The same argument, together with our assertion that the large bump in Fig. 16 is all due to diffuse scattering, leads us to expect that the entire bump will disappear in the presence of helium. That expectation is dramatically confirmed.

In another experiment which was designed to test the intersecting-caustics interpretation of the large nonspecular peak, the heater and bolometer were placed along the $X$ axis as in Fig. 16, but on a longer crystal ( $24 \mathrm{~mm} \times 22 \mathrm{~mm}$ diameter). In this configuration, the caustics do not intersect on the reflection surface, but rather hit the side wall, as illustrated in the inset of Fig. 18; note that there is no large diffuse peak which follows the specular transverse peaks. Since the high-focusing channels for diffuse scattering intersect the side wall, the diffusely scattered phonons arrive at a much earlier time than the specular phonons, which have made two traversals of the full length of the crystal. When the same crystal was cut and repolished, the diffuse-scattering channel intersects the back reflection surface again, and the familiar diffuse bump following the specular channels is recovered, as shown in Fig. 19. Note that the specular peaks, although shifted to diffe rent arrival times, are essentially similar in the two experiments; only the diffuse scattering is strongly affected by the shape of the crystal.

The analysis of caustics and the experimental results presented above have several important implications for the proper interpretation of pho-

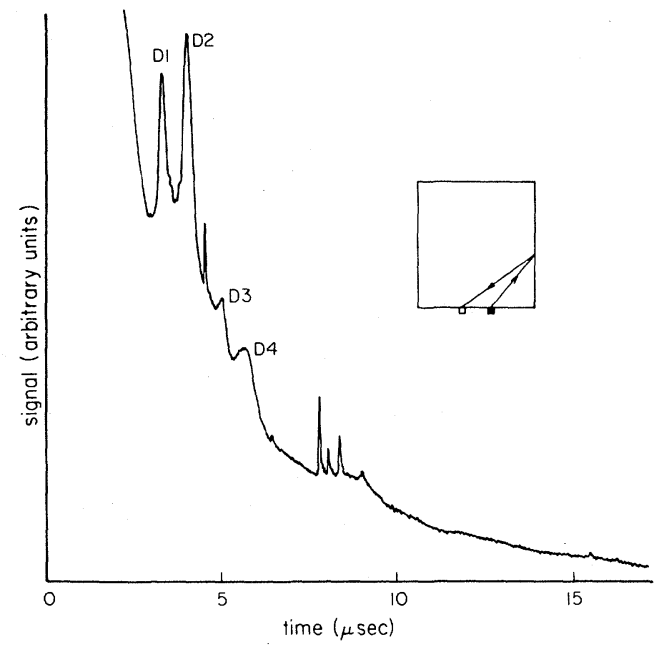

FIG. 18. Reflection signal for heater and bolometer displaced along $X$ axis (as in Fig. 16) but in a crystal 24 $\mathrm{mm} \times 22 \mathrm{~mm}$ diameter. The heater and bolometer caustics intersect in several places on the side wall of the crystal, giving rise to the diffuse peaks $D 1, D 2, D 3$, and $D 4$. The three sharp peaks at $7.5 \mu$ sec are due to specular transverse processes; no large diffuse bump follows these peaks. 


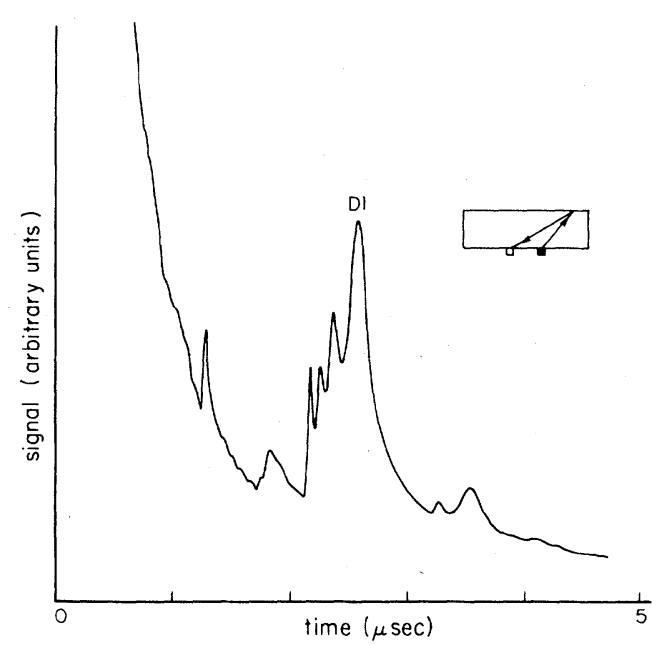

FIG. 19. Reflection signal for precisely the same geometry as in Fig. 18, except the crystal has been cut to a length of $6.9 \mathrm{~mm}$. The caustics intersect on the reflection surface and cause the diffuse bump $D 1$.

non-reflection experiments. Perhaps the most important conclusion is that the diffuse-scattering signal behaves in two essentially different ways depending on whether the heater and bolometer caustics intersect or not. If the caustics do not intersect, the anisotropic emission of energy from the heater is washed out by diffuse reflection, and the signal can be adequately described using an isotropic model as we have done in Sec. II. If the caustics intersect, the diffuse scattering produces rather sharp features which in the past may have been confused with specular peaks. The intersection or nonintersection of the caustics depends critically on the relative orientation of the heater and detector and the shape of the crystal. These parameters have been given insufficient attention by previous investigators. Many experiments have been reported in which the crystallographic plane of the reflection surface is specified, but the plane which contains the heater-detector-surface normal is not. Even if the heater-detector separation is small, changes in the relative orientation with respect to the crystal axes can completely change the received signal, and the apparent effect of helium.

It is interesting to note that the time-of-flight of the diffuse peaks can be predicted from the caustic structure of a point source alone; regions where the focusing factor is finite do not seem to contribute significantly to the diffuse peak. The published tables and plots ${ }^{10,21}$ of average phonon intensities computed on a grid are misleading in the sense that they do not reveal the existence of the caustics. Instead, there are regions of elevated intensity, some of which conceal underlying caustics and contribute heavily to the experimental signal, and others which do neither. Thus, while the old phonon-focusing calculations can be helpful in interpreting certain simple cases,$^{3}$ they cannot explain the details of the experiments described above.

In addition to its utility in predicting experimental results, the geometric theory of focusing developed here also provides an appealing theoretical framework for the discussion of phonon propagation in crystals. Although the detailed calculations are themselves rather involved, catastrophe theory provides a means of extracting a simple and general picture: from a point acoustic source in a crystal there emanate topologically conical caustic surfaces (fold catastrophes) with occasional higher-order line singularities in those surfaces (cusp catastrophes). On the caustics the field falls off more slowly than (distance) ${ }^{-1}$, and on the cusps more slowly still, but it always remains finite and calculable, as it must.

\section{SUMMARY AND CONCLUSIONS}

In this paper, we have discussed the effect of diffuse scattering of phonons at an interface on phonon-reflection spectra. In the absence of strong focusing effects, a simple isotropic theory accounts for the observed line shapes. On the other hand, if the heater-detector-crystal geometry is such that heater and detector caustics intersect (especially near a cusp) on the reflecting surface, almost nothing else is important in understanding the diffuse part of the reflected signal. When helium is present at the interface, the phonons that would have been diffusely scattered are transmitted instead, but the specularly reflected part of the spectrum is not observably affected. Thus, it is the diffusely scattered portion of the thermal energy that is responsible for the anomalous Kapitza effect.

The effects described in the paragraph above could not have been detected without the high-resolution time-of-flight spectra we have obtained and presented, nor could they have been understood without the new theory of phonon focusing we have presented. It is important to realize, however, that although we have isolated part of the cause of the Kapitza effect, the effect is still not understood. We cannot be sure even why part of the energy is diffusely scattered, much less why that part of the energy transmits easily through the interface.

Specular reflection can be understood to be a consequence of the translational invariance of 
the interface parallel to itself. The cause of nonspecular reflection must, therefore, lie in whatever lifts the translational invariance. Obvious candidates are surface roughness, imperfections such as dislocations near the surface, and patches of adsorbed impurities on the surface. Consider surface roughness as an example. If a surface is corrugated with a wavelength larger than that of the incident phonons, the phonons will be diffracted rather than reflected. The optically polished surfaces used in our experiments have a characteris tic roughness scale of approximately $25 \mathrm{~nm}$. The thermal phonons in the heat pulses we used have an energy distribution that peaks at around $25 \mathrm{~nm}$. Thus, one might expect that about half the energy was in the form of long-wavelength phonons for which the surface is flat, and which are, therefore, reflected specularly. The remaining shortwavelength phonons are diffracted, and (for some unknown reason) transmitted if helium is present. This argument would account nicely for our observation that $\eta \simeq 0.5$; i.e., we observe that about half the energy is reflected specularly and half diffusely. The same argument would lead one to expect that, if the detector could resolve phonon frequencies, it would be found that low frequencies are concentrated in the specular peaks, while high frequencies are concentrated in the large nonspec- ular bumps, e.g., in Figs. 7, 16, and 19. Experiments to check that idea are planned. However, evidence already exists that high-frequency phonons are principally responsible for the Kapitza effect. $^{2,22}$ Thus, the whole picture hangs together very well, but it still yields no insight into the mechanism of the effect. The theoretical picture of the effects of surface roughness on the Kapitza resistance seems confused. ${ }^{23}{ }^{24}$ A more realistic theory would be helpful in understanding the results of these experiments.

In conclusion, we wish to point out that the insights gained from the work we have presented here should lead to a new generation of useful experiments. For example, we now see how one can design a phonon generator-detector-crystal geometry in order to concentrate the most interesting part of the spectrum, the nonspecular part, into intense bumps, isolated from all specular reflections. With the nonspecular part thus isolated, its behavior can be studied quantitatively and sensitively in ways not previously possible.

\section{ACKNOWLEDGMENT}

This work was supported in part by NSF Grant No. DMR77-00036 and by a grant from the Caltech President's Fund.
${ }^{1}$ P. Taborek and D. Goodstein, J. Phys. C 12,4737 (1979).

${ }^{2}$ J. T. Folinsbee and J. P. Harrison, J. Low Temp. Phys. 32,469 (1978).

${ }^{3}$ R. E. Horstman and J. Wolter, Phys. Lett. $62 \mathrm{~A}, 279$ (1977).

${ }^{4}$ J. S. Buechner, Ph.D. thesis, Brown University, Providence, Rhode Island, 1976 (unpublished).

${ }^{5}$ H. Kinder and W. Dietsche, Phys. Rev. Lett. $\underline{33}, 578$ (1974).

${ }^{6} \mathrm{~J}$. S. Buechner and H. J. Maris, Phys. Rev. Lett. $\underline{34}$, 316 (1975)

${ }^{7}$ A. R. Long, R. A. Sherlock, and A. F. Wyatt, J. Low Temp. Phys. 15, 523 (1974).

${ }^{8} \mathrm{~J}$. Weber, W. Dietsche, and H. Kinder, Phys. Lett. 64A, 202 (1977).

9 J. Weber, W. Sandmann, W. Dietsche, and H. Kinder, Phys. Rev. Lett. 40, 1469 (1978).

${ }^{10} \mathrm{~B}$. Taylor, H. J. $\bar{M}$ aris, and C. Elbaum, Phys. Rev. B 3, 1426 (1970).

${ }^{11}$ H. J. Maris, J. Acoust. Soc. Am. 50, 812 (1970).

${ }^{12}$ R. J. Von Gutfeld, in Physical Acoustics, edited by W. P. Mason (Academic, New York, 1968), Vol. V, p. 233.
${ }^{13} \mathrm{P}$. Taborek and D. Goodstein, in Phonon Scattering in Condensed Matter, edited by H. J. Maris (Plenum, New York, 1980), p. 215.

${ }^{14} \mathrm{P}$. Taborek and D. Goodstein, Solid State Commun. $\underline{33}$, 1191 (1980).

${ }^{15}$ V. T. Buchwald, Proc. R. Soc. A253, 563 (1959).

${ }^{16}$ M. J. Lighthill, Philos. Trans. R. Soc. A252, 397 (1960).

${ }^{17}$ J. N. L. Connor, Mol. Phys. 31, 33 (1975).

${ }^{18}$ M. V. Berry, Adv. Phys. 25, 1 (1975).

${ }^{19} \mathrm{Cusp}$ points on a caustic should not be confused with cusps on the so-called wave surface.

${ }^{20}$ G. A. Northrop and J. P. Wolfe, Phys. Rev. Lett. $\underline{43}$, 1424 (1979); see also J. C. Hensel and R. C. Dynes, ibid. 43, 1033 (1979).

${ }^{21} \mathrm{C}$. G. Winternheimer and A. K. McCurdy, Phys. Rev. B 18,6576 (1978).

${ }^{22} \mathrm{C} . \overline{\mathrm{H}}$. Anderson and E. S. Sabisky, in Physical Acoustics, edited by W. P. Mason and R. N. Thruston (Academic, New York, 1971), Vol. 8, p. 1.

${ }^{23}$ I. N. Adamenko and I. M. Fuks, Zh. Eksp. Teor. Fiz. 59, 2071 (1970) [Sov. Phys. - JETP 32, 1123 (1971)]. ${ }^{24}$ W. A. Little, Phys. Rev. 123,1909 (1961). 